\title{
Molecular Investigation of the Transmission Pattern of Brucella suis 3 From Inner Mongolia, China
}

\begin{abstract}
Zhi-guo Liu ${ }^{1,2 t}$, Li-jun Wang ${ }^{3 \dagger}$, Dong-ri Piao ${ }^{1}$, Miao Wang ${ }^{2}$, Ri-hong Liu ${ }^{2}$, Hong-yan Zhao ${ }^{1}$, Bu-yun Cui ${ }^{1}$ and Hai Jiang ${ }^{1,4 *}$

${ }^{1}$ State Key Laboratory for Infectious Disease Prevention and Control, National Institute for Communicable Disease Control and Prevention, Chinese Center for Disease Control and Prevention, Beijing, China, ${ }^{2}$ Inner Mongolia Autonomous Region Center for Comprehensive Disease Control and Prevention, Huhhot, China, ${ }^{3}$ Hulun Buir People's Hospital of the Inner Mongolia Autonomous Region, Hohhot, China, ${ }^{4}$ Brucellosis Prevention and Treatment Engineering Technology Research Center of Inner Mongolia Autonomous Regeion, Tongliao, China
\end{abstract}

Brucellosis is an endemic disease in China affecting both humans and livestock. The aim of the present study was to analyze two Brucella strains isolated from sheep spleens from Ulanqab in Inner Mongolia, China using classical and molecular typing techniques. The two strains were identified as Brucella suis biovar 3 and were closely related to isolates previously obtained from two different hosts (human and swine) in Guangxi Province. Our results suggest that $B$. suis can be directly or indirectly transferred from swine to sheep, which act as reservoirs for $B$. suis infection and later transmitted to humans. Multiple locus variable-number tandem repeat analysis (MLVA) is a useful tool for tracing the geographical origin of brucellosis infections and elucidating its transmission patterns.

Keywords: transmission pattern, Brucella suis 3, MLVA, molecular investigation, inner mongolia

\section{BACKGROUND}

*Correspondence: Hai Jiang jianghai@icdc.cn

†These authors have contributed equally to this work

Specialty section:

This article was submitted to Veterinary Infectious Diseases, a section of the journal Frontiers in Veterinary Science

Received: 22 October 2017 Accepted: 10 October 2018 Published: 29 October 2018

Citation:

Liu Z, Wang L, Piao D, Wang M, Liu R, Zhao H, Cui B and Jiang H (2018) Molecular Investigation of the Transmission Pattern of Brucella suis 3 From Inner Mongolia, China. Front. Vet. Sci. 5:271 doi: 10.3389/fvets.2018.00271
Brucellosis is a zoonotic bacterial disease caused by Brucella spp., a gram-negative bacteria that infects a wide range of mammals, including domestic and wild animals and humans, resulting in severe economic loss $(1,2)$. Brucella melitensis, Brucella abortus, and Brucella suis have been reported to infect both animals and humans (3). Infected domestic and wild animals serve as the natural reservoir and source of infection, which occurs through direct contact or consumption of undercooked meat and other contaminated animal products (4).

Prevention of human brucellosis largely depends on disease control in animals (5). Upon isolation, it is essential to identify the Brucella species and biovar, which in turn are utilized in epidemiological follow-ups and control of disease. Identification of Brucella strains in livestock is also essential to importation (6). Classical biotyping methods remain the definitive diagnostic approach and gold standard for confirming and identifying Brucella spp. at the species and biovar levels (7), although it only provides limited epidemiological information. Due to technical difficulties, previous studies have mainly concentrated on Brucella infection surveillance in Inner Mongolia of China, and investigations on the biological significance of shifts in B. suis hosts are limited. The multiple locus variable-number tandem repeat analysis-16 (MLVA-16) scheme has been extensively utilized in resolving closely related isolates $(8,9)$. This method has been proven to be effective in generating epidemiological information on Brucella strains, particularly in identifying its geographical source and elucidating transmission patterns $(10,11)$. In the present study, the MLVA-16 scheme was used to characterize two B. suis isolates from the spleens of sheep from Inner Mongolia that were collected in 2017. 


\section{MATERIALS AND METHODS}

\section{Bacterial Culture and Isolation}

Bacteriological analysis was performed at a biosafety level-3 facility at the Chinese Centre for Disease Prevention and Control, National Institute for Communication Disease Prevention and Control. Forty-one sheep spleens were subjected to bacterial culture. Brucella isolates were primarily identified using standard procedures as described elsewhere $(7,12)$. Two Brucella strains (ws151 and ws152) were isolated from the spleens of sheep from a slaughterhouse in 2016. Brucella suis 1330 was used as a biotyping control.

\section{Biotyping}

All isolates were identified as Brucella species on the basis of classical identification procedures (13), including CO2 requirement and $\mathrm{H} 2 \mathrm{~S}$ production, inhibition of growth by basic fuchsin $(20 \mu \mathrm{g} / \mathrm{mL})$ and thionin $(20 \mu \mathrm{g} / \mathrm{mL})$, agglutination with monospecific antiserum for $\mathrm{A}$ and $\mathrm{M}$ antigens, and phage identification. Brucella monospecific antisera $A$ and $M$ were obtained from the Chinese Centre for Disease Prevention and Control, National Institute for Communication Disease Prevention and Control.

\section{DNA Preparation and MLVA Genotyping}

A loopful of cultured bacterial cells was dissolved in double distilled water, and heat treated at $99^{\circ} \mathrm{C}$ for $20 \mathrm{~min}$. Total genomic DNA was extracted using a bacterial DNA purification kit (Wizard Genomic DNA Purification Kit, Promega, USA), according to the manufacturer instructions and stored at $-20^{\circ} \mathrm{C}$ until analysis. The two isolates were identified on the basis of the Suis-ladder PCR (14). Genotyping was performed using a combination of both minisatellite and microsatellite tandem repeats as previously described $(11,15,16)$.

\section{Analysis of MLVA Data}

Sixteen VNTRs were divided into three panels (panels 1, 2A, and $2 \mathrm{~B}$ ) such as MLVA8 = panel 1 , MLVA11 = panels $1+$ $2 \mathrm{~A}$, MLVA16 $=$ panels $1+2 \mathrm{~A}+2 \mathrm{~B}$. The total number of repeat units at each locus was determined based on amplicon size as previously described (15). The copy number at each locus and each panel was managed as a character dataset using BioNumerics version 6.6 (Applied Maths, Belgium).
Cluster analysis was based on categorical coefficients and the unweighted-pair group method with arithmetic mean algorithm (UPGMA). MLVA-16 was used to compare the epidemiological relationships between strains of $B$. suis biovar 3 obtained from Ulanqab and 59 B. suis isolates from different geographical regions in China (Table $\mathbf{S 1}$ ). The MLVA-16 primers used in this study are presented in Table S2. MLVA profiles of two strains in this study have been submitted to the MLVA bank 2016. (http:// microbesgenotyping.i2bc.paris-saclay.fr/databases).

\section{RESULTS}

In the present study, two Brucella strains were isolated from Ulanqab of Inner Mongolia in 2016. Colony morphology, staining, growth features, and slide agglutination with monospecific anti-Brucella sera were used to characterize the two isolates (Table 1). Using standard bacteriological procedures, the isolates were classified at the species and biovar levels. The profiles of the two isolated strains matched that of $B$. suis, and classical biotyping indicated that the $B$. suis isolate was of biovar 3. Two B. suis biovar 3 strains in this study displayed the same Suis-ladder PCR profiles as that in the corresponding reference strains. Test isolates were further examined by MLVA (Table 2). Two B. suis 3 isolates exhibited similar genetic features, namely, MLVA-8 [4 (2-3-4-11-3-1-5-2)] and MLVA11[31(2-3-4-11-3-1-5-2-4-38-9)], respectively. A comparison of the Ulanqab $B$. suis isolates with the five $B$. suis standard reference strains and vaccine S2 by MLVA- 8 was performed. The Ulanqab B. suis 3 isolates and $B$. suis biovar 3 reference strains had identical MLVA-8 [4 (2-3-4-11-3-1-5-2)] genotypes, and four loci that differed from those of B. suis vaccine S2. The MLVA-11 data were used to trace the geographical source of the test strains comprising human and animal isolates from China. B. suis isolates, which exhibited identical MLVA-11 genotypes with strains from four different provinces, namely, Guangxi $(n=8)$, Guangdong $(n=2)$, Hainan $(n=1)$, Beijing $(n=1)$, respectively, of which of two $B$. suis isolated (bru073, bru074) from Guangxi Province were isolated from human and swine, respectively. MLVA-16 showed that these four strains were (ws151, ws152 and bru073, and bru074) different but had closely related genotypes (Figure 1). MLVA analysis suggested that the isolates from Ulanqab and Guangxi Provinces were derived from

TABLE 1 | Results of classical biotyping of reference and test strain.

\begin{tabular}{|c|c|c|c|c|c|c|c|c|c|c|c|c|}
\hline \multirow[t]{2}{*}{ Strain } & \multicolumn{2}{|c|}{ Growth feature } & \multicolumn{2}{|c|}{ Dye inhibition test } & \multicolumn{4}{|c|}{ Serum agglutination test } & \multicolumn{3}{|c|}{ Phage lysis typing (RTD) } & \multirow[t]{2}{*}{ Result } \\
\hline & $\begin{array}{l}\qquad \mathrm{CO}_{2} \\
\text { requirement }\end{array}$ & $\begin{array}{c}\qquad \mathrm{H}_{2} \mathrm{~S} \\
\text { production }\end{array}$ & Thionin & $\begin{array}{c}\text { basic } \\
\text { fuchsin }\end{array}$ & $\begin{array}{c}\text { Positive } \\
\text { agglutination }\end{array}$ & A & $\mathbf{M}$ & $\mathbf{R}$ & $\mathrm{Tb}$ & $\mathrm{BK}_{2}$ & $\mathbf{W b}$ & \\
\hline BM 16M & - & - & + & + & + & - & + & - & - & + & - & BM bv. 1 \\
\hline BA 544 & \pm & + & - & + & + & + & - & - & + & + & + & BA bv. 1 \\
\hline BS 1330 & - & ++ & + & - & + & + & - & - & - & + & + & BS bv. 1 \\
\hline WS151 & - & - & + & + & + & + & - & - & - & + & + & BS bv. 3 \\
\hline WS152 & - & - & + & + & + & + & - & - & - & + & + & BS bv. 3 \\
\hline
\end{tabular}

BM, Brucella melitensis; BA, Brucella abortus; BS, Brucella suis. 
TABLE 2 | Sixteen variable number of tandem repeat loci of two Ulanqab B. suis genotypes (WS151, WS152).

\begin{tabular}{|c|c|c|c|c|}
\hline \multirow[t]{2}{*}{ Panel } & \multirow[t]{2}{*}{ Locus } & \multicolumn{2}{|c|}{ B. suis biovar 3} & \multirow{2}{*}{$\begin{array}{l}\text { B. suis biovar } 3 \\
\text { reference strains }\end{array}$} \\
\hline & & WS151 & WS152 & \\
\hline \multirow[t]{8}{*}{ Panel 1} & Bruce 06 & 2 & 2 & 2 \\
\hline & Bruce 08 & 3 & 3 & 3 \\
\hline & Bruce 11 & 4 & 4 & 4 \\
\hline & Bruce 12 & 11 & 11 & 11 \\
\hline & Bruce 42 & 3 & 3 & 3 \\
\hline & Bruce 43 & 1 & 1 & 1 \\
\hline & Bruce 45 & 5 & 5 & 5 \\
\hline & Bruce 55 & 2 & 2 & 2 \\
\hline \multirow[t]{8}{*}{ Panel 2} & Bruce 18 & 4 & 4 & 4 \\
\hline & Bruce 19 & 38 & 38 & 38 \\
\hline & Bruce 21 & 9 & 9 & 9 \\
\hline & Bruce 04 & 9 & 8 & 7 \\
\hline & Bruce 07 & 5 & 5 & 5 \\
\hline & Bruce 09 & 7 & 7 & 10 \\
\hline & Bruce 16 & 8 & 8 & 4 \\
\hline & Bruce 30 & 6 & 3 & 5 \\
\hline
\end{tabular}

Detailed information on the B. suis biovar 3 reference strains. Key: REF 686, host: swine, location: United States.

a common origin and were thus closely related. Comparison of the two B. suis isolates of this study with strains from the MLVA bank identified unique genotypes.

\section{DISCUSSION}

The present study isolated and characterized B. suis biovar 3 isolates from sheep spleens from Inner Mongolia. The detection of Brucella species is essential to the the establishment of pathogen prevalence and disease risk. Inner Mongolia has the top three incidence rate of Brucellosis among Chinese provinces. B. melitensis biovar 3 accounts for more than $85 \%$ of the total number of examined isolates in the area. Although $B$. suis biovar 3 has been previously reported as the causative pathogen in human brucellosis in Hainan Province (17), no cases of $B$. suis biovar 3 infection in animals or humans were observed in Inner Mongolia in past three decades. Brucella species are phylogenetically closely related (18), and despite differences in host preference, some species are capable of cross-species infections. Consequently, the findings of this study may be beneficial in better understanding B. suis transmission patterns between humans and different domestic animals.

In the present study, we used biotyping and MLVA methods to analyze Brucella isolates from sheep in Ulanqab of Inner Mongolia. Although classical biotyping is the gold standard for species and biovar attribution of isolates, it does not accurately identify the species and biovar of atypical Brucella spp. (19). MLVA was able to discriminate biovars of most Brucella species and elucidate the relationship among Brucella isolates. However, due to the high genetic diversity of Brucella spp., no single specific method can discriminate all biovars of Brucella spp. (16). Therefore, we suggest that classical biotyping and MLVA characterization be utilized as complementary tools for Brucella strain identification and epidemiological analysis.

The two $B$. suis isolates and $B$. suis reference strain biovar 3 shared panel 1 genotype 4 and were distinguished from other B. suis reference strains (biovars 1, 2, 4, and 5), differing in one to four loci. Sheep in Inner Mongolia receive the S2 vaccine for 3 consecutive years as part of the national brucellosis control program. Jiang et al. reported that vaccine S2 is genetically highly stable when the strain is passaged 40 times in vitro (20). Furthermore, two isolates displayed Suisladder PCR profiles that were similar to that of the B. suis biovar 3 reference strain. This data further confirms that the $B$. suis isolate in this study is distinct from that used in the S2 vaccine.

MLVA-11 has been utilized in tracing the geographical origin of brucellosis infections. B. suis biovar 3 isolates in this study have identical MLVA-11 genotypes of strains from four different provinces of China, including Guangxi, Guangdong, Hainan, and Beijing provinces, suggesting that these were possibly derived from a common origin. A previous study confirmed that $B$. suis biovar 3 has only been recently isolated in the Guangdong and Hainan provinces (17). However, brucellosis epidemics involving $B$. suis biovar 3 exclusively occurred in Hainan Province. Furthermore, we observed that B. suis biovar 3 strains from the north (Inner Mongolia and Beijing) and south (Guangdong and Guangxi) of China were of MLVA-11 genotype 31 , despite originating from different hosts, including humans, swine, and sheep. These data reveal that B. suis biovar 3 strains are continuously circulating among different hosts in brucellosis epidemic regions in China.

Previous studies have confirmed the correlation between MLVA-16 genotyping results with epidemiological data, particularly those displaying identical or very closely related genotypes $(8,11,16,21,22)$. The epidemiological relationship among isolates from different geographical origins in China was evaluated using the MLVA-16 scheme. Two B. suis biovar 3 strains from this study $(n=2)$ shared a similar MLVA16 genotype with strains obtained from Guangxi Province $(n=2)$ and mainly differed in three highly variable loci (bruce04, bruce16, and bruce30) in Panel 2B. They could also be distinguished from most of the other strains from China, thereby suggesting a potential epidemiological correlation among the isolates. The three-locus differencein the highly variable microsatellites from panel $2 \mathrm{~B}$ reveals that these strains are closely related and presumably originated from a recent common ancestor.

A previous study has reported that the phenomenon of host shifting is uncommon between $B$. suis (swine) and accessory hosts (e.g., sheep, cattle, and spotted deer) (20), although only focusing on brucellosis serology and epidemiology. The present study described the first isolation of $B$. suis biovar 3 from sheep spleens in Inner Mongolia, thereby confirming that $B$. suis biovar 3 is capable of shifting hosts between swine and sheep. The findings 


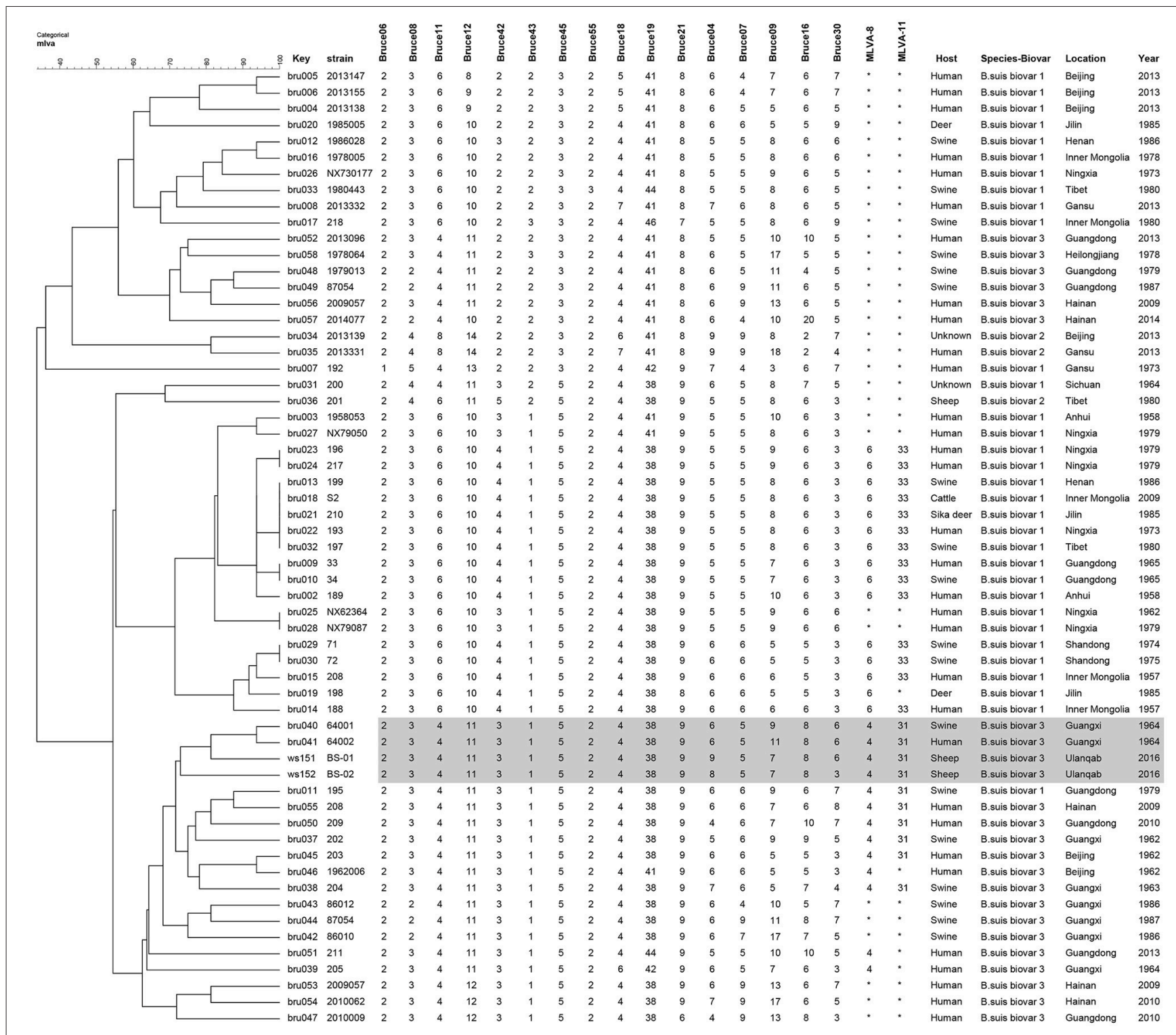

FIGURE 1 | Dendrogram based on the MLVA-16 genotyping assay showing the relationship between the two B. suis isolates in this study and 67 other B. suis biovars from China. The rows highlighted in gray indicate that these have similar MLVA-16 genotypes.

of the present study suggest that $B$. suis biovar 3 continues to circulate in brucellosis endemic regions with swine and sheep as reservoir hosts and subsequently transmitted to other animals and humans.

\section{CONCLUSIONS}

This is the first study that has characterized B. suis biovar 3 isolated from sheep spleens in Inner Mongolia that had the MLVA-16 genotype that was similar to strains isolated from different hosts (human and swine) in Guangxi Province. Information on the prevalence and circulation of Brucella strains in different livestock species facilitates in understanding its transmission patterns and risk factors for infection. The present study highlights that B. suis is capable of host shifts between swine and sheep, eventually infecting humans. MLVA has been utilized to elucidate the biological significance of $B$. suis host shifts between swine and sheep. We suggest that $B$. suis be considered as a significant zoonotic risk to humans and animals in this region and additional strains from wildlife be isolated and characterized.

\section{AUTHOR CONTRIBUTIONS}

ZL performed strain isolation, MLVA typing and cluster analysis, and drafted the manuscript; $M W$ and LW conducted epidemiological investigations and data analysis; $\mathrm{HZ}$ and 
DP prepared the DNA samples; HJ participated in the design of the study and critically reviewed the manuscript; $\mathrm{BC}$ and $\mathrm{RL}$ participated in the design of the study and also managed the project. All authors have read and approved the final manuscript.

\section{FUNDING}

This study was supported by Natural Science Fund of Inner Mongolia Autonomous Region of China (Grant No. 2018MS08004). The funding agency had no role in the study design, data collection and analysis, decision to publish, or preparation of the manuscript.

\section{REFERENCES}

1. Ferreira AC, Chambel L, Tenreiro T, Cardoso R, Flor L, Dias IT, et al. MLVA16 typing of Portuguese human and animal Brucella melitensis and Brucella abortus isolates. PLoS ONE (2012) 7:e42514. doi: 10.1371/journal.pone.0042514

2. Silva TM, Costa EA, Paixao TA, Tsolis RM, Santos RL. Laboratory animal models for brucellosis research. J Biomed Biotechnol. (2011) 2011:518323. doi: $10.1155 / 2011 / 518323$

3. Godfroid J, Scholz HC, Barbier T, Nicolas C, Wattiau P, Fretin D, et al. Brucellosis at the animal/ecosystem/human interface at the beginning of the 21st century. Prev Vet Med. (2011) 102:118-31. doi: 10.1016/j.prevetmed.2011.04.007

4. Ravanel N, Gestin B, Maurin M. In vitro selection of fluoroquinolone resistance in Brucella melitensis. Int J Antimicrob Agents. (2009) 34:76-81. doi: 10.1016/j.ijantimicag.2009.01.002

5. De Massis F, Petrini A, Giovannini A. Reliability evaluation of sampling plan fixed by Council Directive 91/68/EEC for the maintenance of officially brucellosis-free flock status. J Vet Med B Infect Dis Vet Public Health (2005) 52:284-90. doi: 10.1111/j.1439-0450.2005.00856.x

6. Di Giannatale E, De Massis F, Ancora M, Zilli K, Alessiani A. Typing of Brucella field strains isolated from livestock populations in Italy between 2001 and 2006. Vet Ital. (2008) 44:383-8.

7. De Massis F, Ancora M, Atzeni M, Rolesu S, Bandino E, Danzetta ML, et al. MLVA as an epidemiological tool to trace back brucella melitensis biovar 1 re-emergence in Italy. Transbound Emerg Dis. (2015) 62:463-9. doi: $10.1111 /$ tbed. 12397

8. Allen A, Breadon E, Byrne A, Mallon T, Skuce R, Groussaud P, et al. Molecular epidemiology of Brucella abortus in Northern Ireland-1991 to 2012. PLoS ONE (2015) 10:e136721. doi: 10.1371/journal.pone.0136721

9. Shevtsov A, Ramanculov E, Shevtsova E, Kairzhanova A, Tarlykov P, Filipenko M, et al. Genetic diversity of Brucella abortus and Brucella melitensis in Kazakhstan using MLVA-16. Infect Genet Evol. (2015) 34:173-80. doi: 10.1016/j.meegid.2015.07.008

10. Mathew C, Stokstad M, Johansen TB, Klevar S, Mdegela RH, Mwamengele G, et al. First isolation, identification, phenotypic and genotypic characterization of Brucella abortus biovar 3 from dairy cattle in Tanzania. Bmc Vet Res. (2015) 11:156. doi: 10.1186/s12917-015-0476-8

11. Liu ZG, Di DD, Wang M, Liu RH, Zhao HY, Piao DR, et al. MLVA genotyping characteristics of human brucella melitensis isolated from ulanqab of inner mongolia, china. Front. Microbiol. (2017) 8:6. doi: 10.3389/fmicb.2017.00006

12. Al Dahouk S, Tomaso H, Nockler K, Neubauer H, Frangoulidis D. Laboratory-based diagnosis of brucellosis-a review of the literature. Part II: serological tests for brucellosis. Clin Lab. (2003) 49:577-89.

13. Alton GG, Jones LM, Pietz DE. Laboratory techniques in brucellosis. Monogr Ser World Health Organ. (1975) 55:1-163.

\section{ACKNOWLEDGMENTS}

The authors thank the staff of the Brucellosis Clinic, Ulanqab Centre for Endemic Disease Prevention for providing information on sample collection. The authors thank ACCDON (www.Accdon.com) for linguistic assistance during the preparation of this manuscript.

\section{SUPPLEMENTARY MATERIAL}

The Supplementary Material for this article can be found online at: https://www.frontiersin.org/articles/10.3389/fvets. 2018.00271/full\#supplementary-material

14. Lopez-Goni I, Garcia-Yoldi D, Marin CM, de Miguel MJ, Barquero-Calvo E, Guzman-Verri C, et al. New bruce-ladder multiplex PCR assay for the biovar typing of Brucella suis and the discrimination of Brucella suis and Brucella canis. Vet Microbiol. (2011) 154:152-5. doi: 10.1016/j.vetmic.2011. 06.035

15. Le Fleche P, Jacques I, Grayon M, Al DS, Bouchon P, Denoeud F, et al. Evaluation and selection of tandem repeat loci for a Brucella MLVA typing assay. BMC Microbiol. (2006) 6:9. doi: 10.1186/1471-2180-6-9

16. Al Dahouk S, Fleche PL, Nockler K, Jacques I, Grayon M, Scholz HC, et al. Evaluation of Brucella MLVA typing for human brucellosis. J Microbiol Methods (2007) 69:137-45. doi: 10.1016/j.mimet.2006. 12.015

17. Jiang H, Chen H, Chen JD, Tian GZ, Zhao HY, Piao DR, et al. (2012) Genetic comparison of Brucella suis biovar 3 in clinical cases in China. Vet Microbiol. 160:546-8. doi: 10.1016/j.vetmic.2012.06.030

18. Scholz HC, Vergnaud G. Molecular characterisation of Brucella species. Rev Sci Tech. (2013) 32:149-62. doi: 10.20506/rst.32.1.2189

19. Marianelli C, Graziani C, Santangelo C, Xibilia MT, Imbriani A, Amato R, et al. Molecular epidemiological and antibiotic susceptibility characterization of Brucella isolates from humans in Sicily, Italy. J Clin Microbiol. (2007) 45:2923-8. doi: 10.1128/JCM.00822-07

20. Jiang $\mathrm{H}$, Wang $\mathrm{H}, \mathrm{Xu} \mathrm{L}, \mathrm{Hu}$ G, Ma J, Xiao P, et al. MLVA genotyping of Brucella melitensis and Brucella abortus isolates from different animal species and humans and identification of Brucella suis vaccine strain S2 from cattle in China. PLoS ONE 8:e76332. doi: 10.1371/journal.pone.00 76332

21. Kilic S, Ivanov IN, Durmaz R, Bayraktar MR, Ayaslioglu E, Uyanik MH, et al. Multiple-locus variable-number tandem-repeat analysis genotyping of human Brucella isolates from Turkey. J Clin Microbiol. (2011) 49:3276-83. doi: 10.1128/JCM.02538-10

22. Nockler K, Maves R, Cepeda D, Draeger A, Mayer-Scholl A, Chacaltana J, et al. Molecular epidemiology of Brucella genotypes in patients at a major hospital in central Peru. J Clin Microbiol. (2009) 47:3147-55. doi: 10.1128/JCM.00900-09

Conflict of Interest Statement: The authors declare that the research was conducted in the absence of any commercial or financial relationships that could be construed as a potential conflict of interest.

Copyright (c) 2018 Liu, Wang, Piao, Wang, Liu, Zhao, Cui and Jiang. This is an open-access article distributed under the terms of the Creative Commons Attribution License (CC BY). The use, distribution or reproduction in other forums is permitted, provided the original author(s) and the copyright owner(s) are credited and that the original publication in this journal is cited, in accordance with accepted academic practice. No use, distribution or reproduction is permitted which does not comply with these terms. 